\title{
Sulforaphane Prevents Neuronal Apoptosis and Memory Impairment in Diabetic Rats
}

\author{
Gengyin Wang ${ }^{\mathrm{a}}$ Hui Fanga,b Yanfeng Zhen ${ }^{\mathrm{b}}$ Gang Xuc Jinli Tian ${ }^{\mathrm{b}}$ Yazhong Zhang ${ }^{\mathrm{b}}$

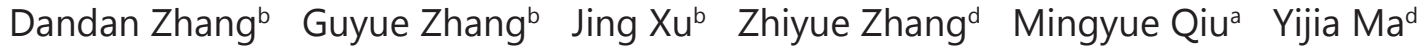 \\ Hongrui Zhange Xinxin Zhang ${ }^{\text {a }}$
}

aDepartment of Internal Medicine, Hebei Medical University, Shijiazhuang, ${ }^{\mathrm{b} S e c o n d}$ Department of Endocrinology, Tangshan Gongren Hospital, Tangshan, 'Department of Burns and Orthopedics,

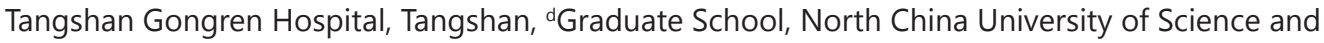
Technology, Tangshan, 'Center of Health Examination, Tangshan People's Hospital, Tangshan, China

\section{Key Words}

Sulforaphane $\cdot$ Diabetes $\bullet$ Memory impairment $•$ Apoptosis

\begin{abstract}
Background/Aims: To explore the effects of sulforaphane (SFN) on neuronal apoptosis in hippocampus and memory impairment in diabetic rats. Methods: Thirty male rats were randomly divided into normal control, diabetic model and SFN treatment groups ( $N=10$ in each group). Streptozotocin (STZ) was applied to establish diabetic model. Water Morris maze task was applied to test learning and memory. Tunel assaying was used to detect apoptosis in hippocampus. The expressions of Caspase- 3 and myeloid cell leukemia $1(\mathrm{MCL}-1)$ were detected by western blotting. Neurotrophic factor levels and AKT/GSK3 $\beta$ pathway were also detected. Results: Compared with normal control, learning and memory were apparently impaired, with up-regulation of Caspase- 3 and down-regulation of $\mathrm{MCL}-1$ in diabetic rats. Apoptotic neurons were also found in CA1 region after diabetic modeling. By contrast, SFN treatment prevented the memory impairment, decreased the apoptosis of hippocampal neurons. SFN also attenuated the abnormal expression of Caspase- 3 and $\mathrm{MCL}-1$ in diabetic model. Mechanically, SFN treatment reversed diabetic modeling-induced decrease of $p$-Akt, p-GSK3 $\beta$, NGF and BDNF expressions. Conclusion: SFN could prevent the memory impairment and apoptosis of hippocampal neurons in diabetic rat. The possible mechanism was related to the regulation of neurotropic factors and Akt/GSK3 $\beta$ pathway.

\section{Introduction}

Diabetes mellitus (DM) was a metabolism disease characterized by insulin resistance and related decrease of insulin release [1]. Many complications were found in diabetic patients, including brain abnormality. Clinically, the ability of learning and memory, the 


\section{Cellular Physiology Cell Physiol Biochem 2016;39:901-907

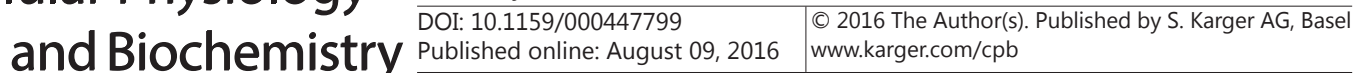 \\ Wang et al.: SFN on Neuronal Apoptosis and Memory Impairment}

space navigation obviously decreased in diabetic patients [2, 3]. Dementia was also found in some severe diabetic cases. Although there was no consistent definition of diabetic and brain disease, electrophysiological and image abnormalities could be used to confirm the chronic mild cognition impairment in diabetic patients [4]. Epidemiologically, the diabetic patients with mild cognition impairment have a high rate to progress into dementia [5]. Nevertheless, the exact pathological mechanisms for cognitive impairment in diabetic patients were still elusive.

Accumulating evidence suggested that neurological diseases involved in diabetes were related to apoptosis of neurons [6]. Loss of neurons contributes to neuronal and circuit dysfunction. Possible mechanisms were related to metabolism dysfunction, vascular factors, and deficient of nerve growth factors. In addition, the blockage of cell survival signaling pathways was also related to neurological diseases regulated by diabetes [7-9].

Sulforaphane (SFN) is a compound within the isothiocyanate group of organosulfur compounds, which widely distributed in cruciferous vegetables such as broccoli, Brussels sprouts or cabbages. SFN has a mount of pharmacological activities, including antioxidative [10], anticancer [11], antibacterial [12] and anti-inflammation [13], etc. Recent studies also suggested that SFN could be a potential compound to enhance memory in disease models [14]. However, their mechanisms were not disclosed. In this study, we aimed to explore whether SFN could prevent memory deficit in diabetic model and its underlying mechanisms.

\section{Materials and Methods}

\section{Experimental animals}

30 sprague-Dawley (SD) rats (male, 12 weeks) in clean grade were obtained from Animal Center of Hebei Medical University (1511040) and raised in the temperature of $25 \pm 2^{\circ} \mathrm{C}$ with free access to food and water. All the experiments were performed with the approval of Ethnics Committee of Hebei Medical University. After one week adaption, 30 rats were randomly divided into three groups: normal control, diabetes mellitus group and SFN treatment groups. $50 \mathrm{mg} / \mathrm{kg}$ streptozotocin (STZ, Sigma) was administrated through intraperitoneal injection to induce diabetic model. Diabetic models were regarded as successful if blood glucose levels were higher than $16.7 \mathrm{mmol} / \mathrm{L}$. 11 weeks after successfully modeling, the rats in SFN group were treated with SFN $(25 \mathrm{mg} / \mathrm{kg}$ ) orally once daily for consecutive 14 days. After treatment, behavioral tests and biochemical experiments were carried out.

\section{Water Morris maze}

Animals were tested by water Morris maze 2 weeks after SFN treatments. At the beginning of place navigation task, rats were placed in a circle pool, allowing them to swim for $5 \mathrm{~min}$. Tests were carried out at a fixed time every day for consecutive 4 days. Video camera tracking system recorded the time it took for rats to find platforms (escape latency) and their swimming routes. During the 4 training sections, rats were placed into the pool at 4 different starting points (different quadrants). If rats found platforms or found different platforms within $120 \mathrm{sec}$ during Morris water maze test (latency is recorded as $120 \mathrm{sec}$ ), they were taken onto platforms, allowing 15 sec's rest before next training section. The average of latency during 4 training sections every day was recorded as the study result of that day. At day 5 of spatial probe, platform was removed, and rats were placed into water at any starting point. All rats were placed at the same starting point, and the time of rats staying in the quadrant where platform was placed was recorded.

\section{Western blot}

After the behavioral tests, the rats were decapitated and brains were obtained. The brain tissue lysates were separated by SDS-PAGE, transferred to PVDF, and immunoblotted using specific antibodies against Caspase-3 (1:1000; Abcam), myeloid cell leukemia 1(MCL-1) (1:1000, Abcam), NGF (1:1000; Abcam), BDNF (1:1000, Abcam), p-Akt (1:1000, Abcam), Akt (1:1000, Abcam), GSK3 $\beta$ (1:1000, Abcam) and p-GSK3 $\beta$ (1:1000, Abcam). The bound antibodies were detected using horseradish peroxidase-conjugated secondary antibodies and an enhanced chemiluminescence detection system. 
Tunel Assay

The apoptosis of hippocampal neurons were detected by Tunel assay. The brain tissue was fixed in 4\% PFA and embedded into paraffin wax. In Situ Cell Death Detection Kit (Roche, Switzerland) was applied to detect the apoptosis according to the manufacturer's instructions. Apoptotic changes were measured via fluorescence microscopy (Olympus, Tokyo, Japan). The images were taken using a light microscope (Olympus, Japan). We referred to the previous publication to analyze the apoptotic rate [15]. The positive rate of $0-1 \%$ was defined as score $0,1-10 \%$ as score $1,10-50 \%$ as $2,50-80 \%$ as 3 and $80-100 \%$ as 4 . The expressions were divided into negative (score 0 ), weak positive (score 1), positive (score 2) and strong positive expressions (score 3), respectively.

\section{Statistical Analysis}

All data were presented as mean \pm SED. Differences among three or more groups were compared by one way analysis of variance (ANOVA), followed by Bonferroni post hoc testing for multiple comparisons. $p$ values of 0.05 or less were regarded as significant.

\section{Results}

SFN prevented the impairments of spatial learning and memory in diabetic model

We next tested the learning and memory in different groups. As shown in Fig. 1A, the latency to find the platform was significantly higher in model group than that in normal control group. By contrast, SFN treatment significantly decreased the latency to find the platform compared with diabetic model group. After four-day training, the platform was removed. Under this condition, the time spent in target quadrant was significantly lower in model group than that in control group (Fig. 1B). By contrast, the time in SFN treatment group was significantly increased compared with model group.

\section{SFN prevented apoptosis of hippocampal neurons in CA1 region}

As shown in Fig. 2, apoptotic neurons were significantly increased in the CA1 region in model group. However, SFN could significantly decrease the numbers of apoptotic neurons in CA1 region.

SFN prevented the abnormalities of Caspase-3 and MCL-1 expressions in diabetic model

We also detected caspase-3 and MCL-1 expressions (Fig. 3). Compared with normal control group, hippocampal Caspase-3 was increased in model group, while it was downregulated by SFN treatment. MCL-1 expression was increased in model group, however decreased by SFN treatment.

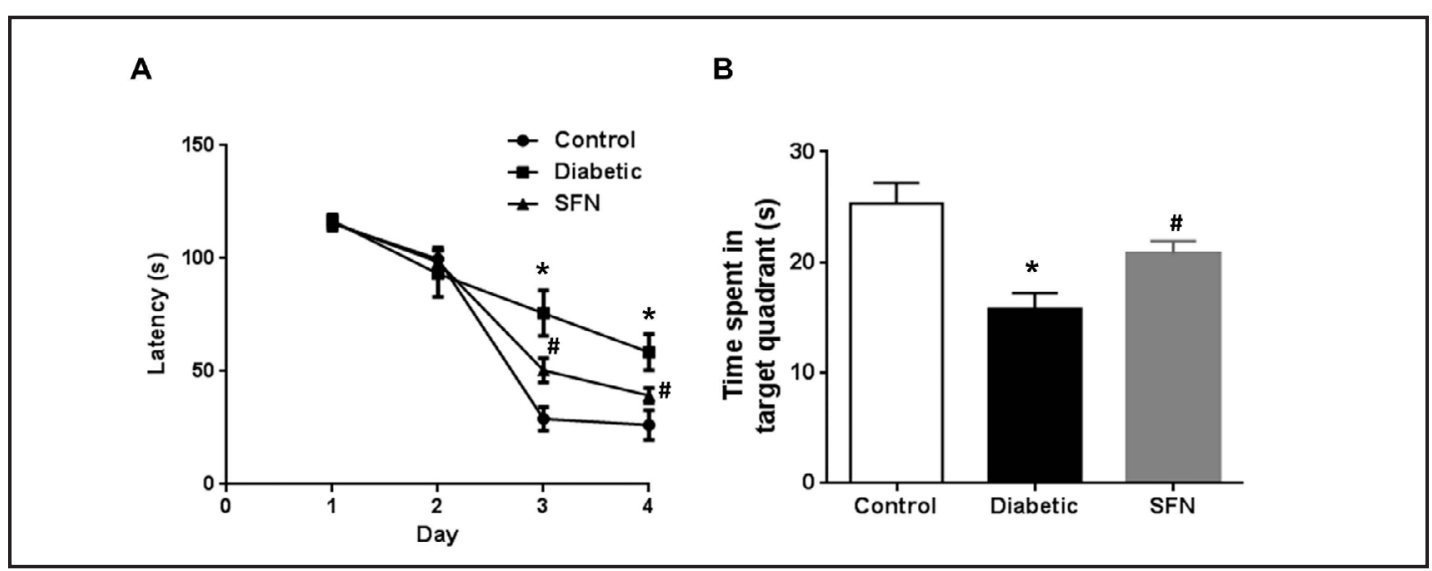

Fig. 1. SFN enhanced learning and memory in diabetic rat model. (A) The latency to find the platform during the 4-day training. (B) The time spent in target quadrant after removal of the platform. $* \mathrm{p}<0.05$ compared with control group. ${ }^{*} \mathrm{p}<0.05$ compared with model group. 
Fig. 2. SFN mitigated the neuronal apoptosis in diabetic rats. (A) A few apoptotic neurons were found in control group. (B) Significant increase of apoptotic neurons were found in diabetic model. (C) The apoptotic neurons were eliminated in SFN treatment group. (D) Quantificaion data of the apoptotic cells. $* \mathrm{p}<0.05$ compared with control group. \# $\mathrm{p}<0.05$ compared with model group.
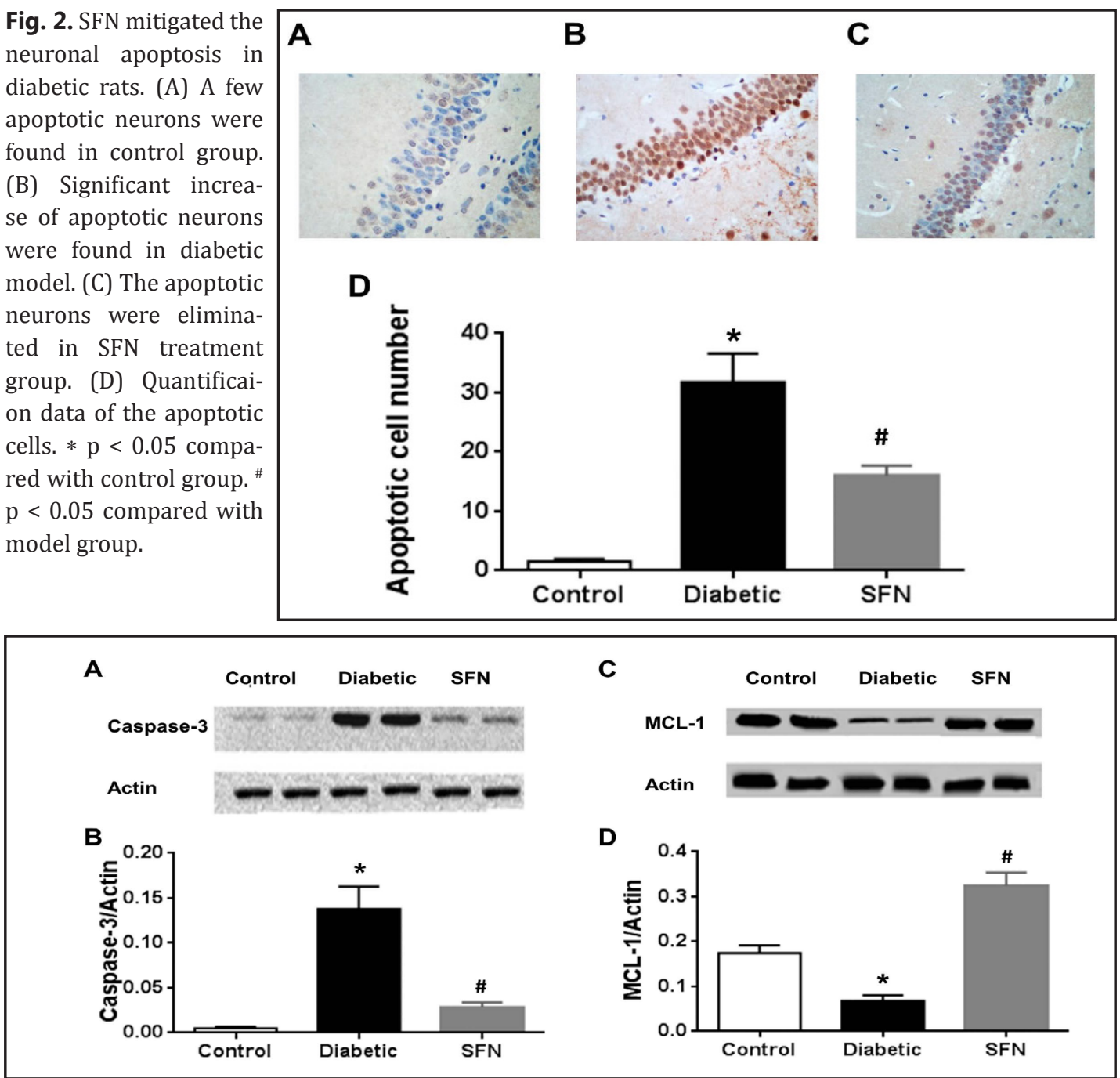

Fig. 3. SFN decreased Caspase-3 and increased MCL-1 expressions in diabetic rats. (A) Representative blots of Caspase-3. (B) Quantified data of Caspase-3. (C) Representative blots of MCL-1. (D) Quantified data of MCL-1. * p $<0.05$ compared with control group. ${ }^{*} \mathrm{p}<0.05$ compared with model group.

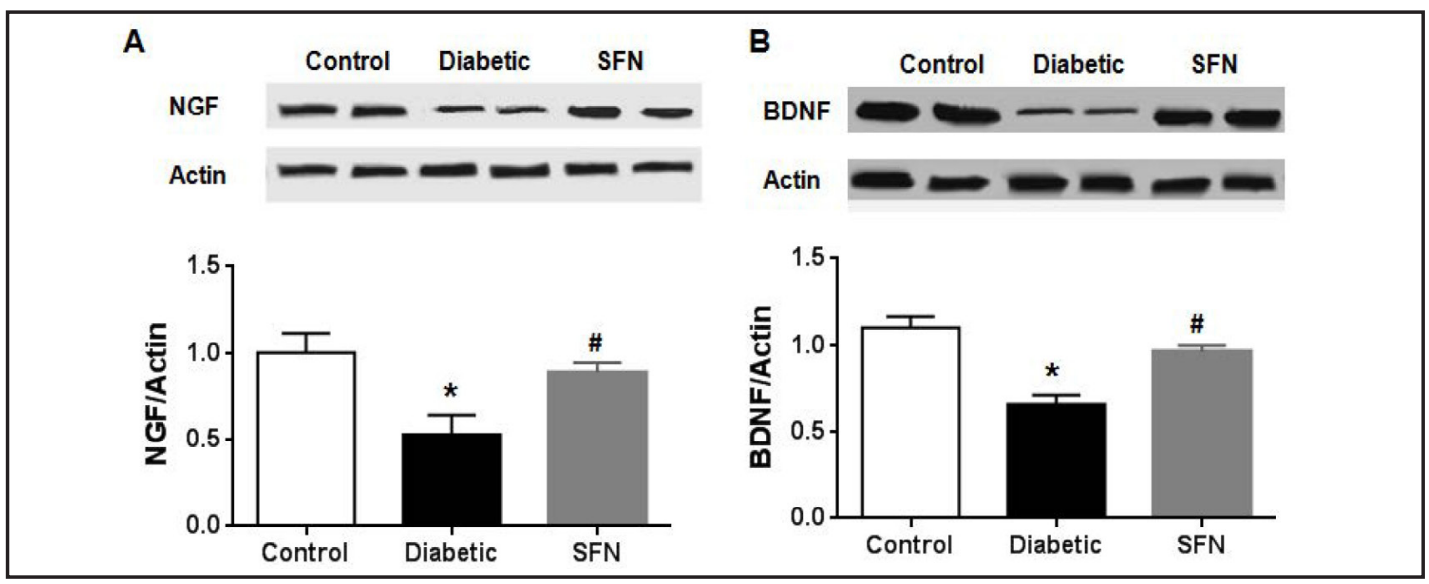

Fig. 4. SFN reversed neurotrophic factors level induced by diabetic modeling. (A) NGF expression; (B) BDNF expression. $* \mathrm{p}<0.05$ compared with control group. ${ }^{*} \mathrm{p}<0.05$ compared with model group. 


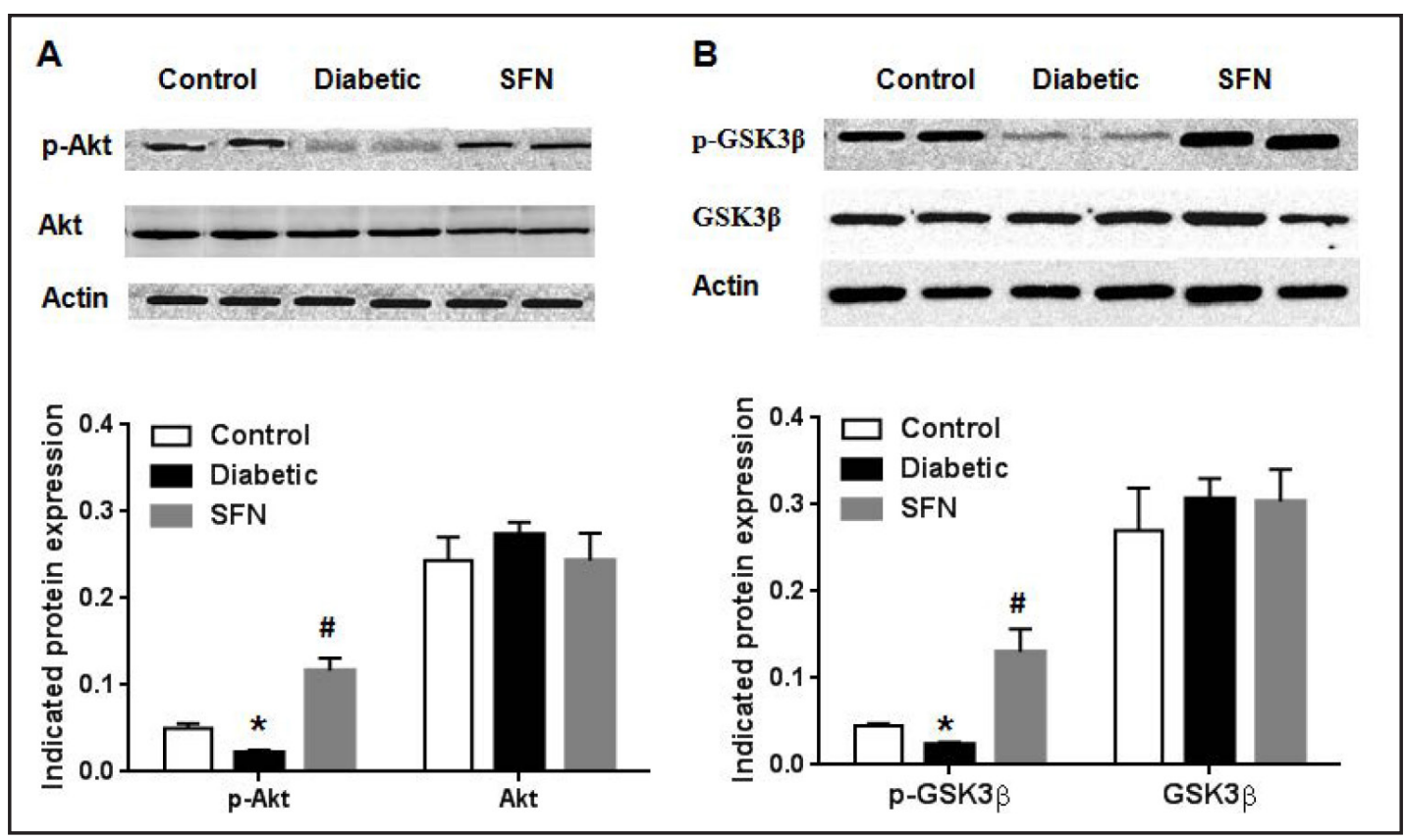

Fig. 5. SFN reversed p-Akt and p-GSK3 $\beta$ expression induced by diabetic modeling. (A) NGF expression; (B) BDNF expression. $* \mathrm{p}<0.05$ compared with control group. ${ }^{*} \mathrm{p}<0.05$ compared with model group.

SFN reversed the decrease of neurotrophic factors

The neurotrophic factors, NGF and BDNF were also detected in different groups. Diabetic modeling decreased both NGF and BDNF expressions. By contrast, SFN treatment could reverse the decrease (Fig. 4).

SFN reversed the deficit of Akt/GSK3 $\beta$ pathway

Akt/GSK3 $\beta$ pathway was supposed as a cell survival pathway. Therefore, we also determined the Akt/GSK3 $\beta$ pathway. As shown in Fig. 5, Diabetic modeling decreased both p-Akt and p-GSK3 $\beta$ expressions. SFN treatment reversed the decrease of p-Akt and p-GSK3 $\beta$ expressions. By contrast, diabetic modeling or SFN treatment did not affect total Akt and GSK3 $\beta$ expressions.

\section{Discussion}

Previous study reported that dietary intake of sulforaphane-rich broccoli sprout extracts during juvenile and adolescence prevented phencyclidine-induced cognitive deficits [16]. That indicated the pharmacological activity of SFN in recovery of memory impairment. SFN is a natural dietary isothiocyanate and was reported to ameliorate memory impairment caused by okadaic acid [17] and scopolamine [18]. SFN also improved cognitive function in traumatic brain injury [19]. In addition, SFN was also reported to ameliorate memory impairment in AD model. In this study, we demonstrated the pharmacological activity of SFN in preventing the memory impairment in diabetic model. This study further evidenced the memory ameliorating effect of SFN in different disease models.

Hippocampus was thought to be an important brain region responsible for memory formation and consolidation [20]. The loss of pyramid neurons in neurological diseases caused deficit of neuronal circuit and impairment of hippocampal synaptic plasticity. As evidenced by previous studies, hippocampal neurons were severely damaged in STZ-induced diabetic models [21]. Moreover, apoptosis was apparently elicited after STZ injection [7]. How STZ caused hippocampal neuronal death was not clear. Intriguingly, there were a lot of 
reports which might represent the mechanisms. On the one hand, neurotrophic factors were decreased after STZ injection. The decrease of neurotrophic factors have been confirmed to regulate the neuronal survival and might partially explain the pyramid neuronal loss [22]. On the other hand, cell survival signaling pathways were also found to be decreased after STZ injection. Typically, Akt and GSK-3 were dephosphorylated in diabetic model [7-9]. The abnormality of those signaling pathways might be potential reasons for the loss of neurons.

Apoptosis is an important type of important programmed cell death, which has been specifically clarified previously. A lot of signaling pathways or organelle damage will elicit apoptosis. Undoubtedly, apoptosis is an important way for pyramid neuron loss. BCL family and caspases are prominent for the apoptosis. MCL-1 is one gene in BCL family, which inhibits caspase-3 and prohibits apoptosis [23]. In our study, we verified that hippocampal MCL1 was down-regulated, while Caspase-3 was increased after diabetic modeling. However, SFN could antagonize the changes of MCL-1 and caspase-3. These data further support that SFN ameliorates the memory impairment in diabetic model through inhibiting apoptosis of hippocampal neurons.

SFN is a kind of isocyanate, mainly from green Cruciferous vegetables like broccoli. SFN possesses a serial of pharmacological activities, such as antioxidant and antitumor. As a chemical preventive medicine, SFN has attracted extensive attention. The compound is known to be an important activator of Nrf2. SFN can induce Nrf2 activation and nucleus translocation, and combines with ARE to regulate the expression of phase II detoxification enzyme to clear ROS. Oxidative stress was thought to be one pathological factor for cell death. The antioxidants, such as grape seed extract and vitamin E were also reported to mitigate hippocampal cell loss in diabetic model [24]. In addition, SFN also possibly ameliorated the memory impairment in diabetic model through anti-inflammation [25]. Recent studies have demonstrated that Nrf2-ARE signaling is also involved in attenuating inflammation-associated pathogenesis [26]. However, these potential reasons still require further verifications.

Neurotrophic factors are important regulators, which not only modulate synaptic transmission or neuronal cell death $[27,28]$. In this study, we found that after diabetic modeling, both of NGF and BDNF were down-regulated, while SFN treatment reversed the down-regulation. PI3K/Akt signaling pathway is a cell survival pathway [28]. In our study, we also found that diabetic modeling decreased the phosphorylation of Akt and GSK3 $\beta$, which were reversed by SFN treatment.

\section{Conclusion}

In this study, we reported that SFN could ameliorate the memory impairment in STZinduced diabetic model. The potential mechanism underlying the protection was through inhibiting caspase- 3 and MCL-1 dependent apoptosis of hippocampal neurons.

\section{Disclosure Statement}

None.

\section{References}

1 American Diabetes A: Standards of medical care for patients with diabetes mellitus. Diabetes Care 2003;26:S33-50.

2 McNay EC, Recknagel AK: Brain insulin signaling: A key component of cognitive processes and a potential basis for cognitive impairment in type 2 diabetes. Neurobiol Learn Mem 2011;96:432-442.

3 Vignini A, Giulietti A, Nanetti L, Raffaelli F, Giusti L, Mazzanti L, Provinciali L: Alzheimer's disease and diabetes: New insights and unifying therapies. Curr Diabetes Rev 2013;9:218-227.

4 Biessels GJ, Staekenborg S, Brunner E, Brayne C, Scheltens P: Risk of dementia in diabetes mellitus: A systematic review. Lancet Neurol 2006;5:64-74. 


\section{Cellular Physiology Cell Physiol Biochem 2016;39:901-907 \begin{tabular}{l|l|l} 
and Biochemistry $\begin{array}{l}\text { DOI: 10.1159/000447799 } \\
\text { Published online: August 09,2016 }\end{array}$ & $\begin{array}{l}\text { (c) } 2016 \text { The Author(s). Published by S. Karger AG, Basel } \\
\text { www.karger.com/cpb }\end{array}$
\end{tabular} \\ Wang et al.: SFN on Neuronal Apoptosis and Memory Impairment}

5 Meneilly GS, Tessier DM: Diabetes, dementia and hypoglycemia. Can J Diabetes 2016;40:73-76.

6 Barber AJ, Gardner TW, Abcouwer SF: The significance of vascular and neural apoptosis to the pathology of diabetic retinopathy. Invest Ophthalmol Vis Sci 2011;52:1156-1163.

7 Sun LJ, Hou XH, Xue SH, Yan F, Dai YJ, Zhao CH, Wang F, Yang RH: Fish oil modulates glycogen synthase kinase-3 signaling pathway in diabetes-induced hippocampal neurons apoptosis. Brain Res 2014;1574:3749.

8 Cui W, Zhang Y, Lu D, Ren M, Yuan G: Upregulation of pakt by glial cell linederived neurotrophic factor ameliorates cell apoptosis in the hippocampus of rats with streptozotocininduced diabetic encephalopathy. Mol Med Rep 2016;13:543-549.

9 Jia D, Heng LJ, Yang RH, Gao GD: Fish oil improves learning impairments of diabetic rats by blocking pi3k/ akt/nuclear factor-kappab-mediated inflammatory pathways. Neuroscience 2014;258:228-237.

10 Danilov CA, Chandrasekaran K, Racz J, Soane L, Zielke C, Fiskum G: Sulforaphane protects astrocytes against oxidative stress and delayed death caused by oxygen and glucose deprivation. Glia 2009;57:645-656.

11 Gamet-Payrastre L: Signaling pathways and intracellular targets of sulforaphane mediating cell cycle arrest and apoptosis. Curr Cancer Drug Targets 2006;6:135-145.

12 Galan MV, Kishan AA, Silverman AL: Oral broccoli sprouts for the treatment of helicobacter pylori infection: A preliminary report. Dig Dis Sci 2004;49:1088-1090.

13 Brandenburg LO, Kipp M, Lucius R, Pufe T, Wruck CJ: Sulforaphane suppresses lps-induced inflammation in primary rat microglia. Inflamm Res 2010;59:443-450.

14 Ping Z, Liu W, Kang Z, Cai J, Wang Q, Cheng N, Wang S, Wang S, Zhang JH, Sun X: Sulforaphane protects brains against hypoxic-ischemic injury through induction of nrf2-dependent phase 2 enzyme. Brain Res 2010;1343:178-185.

15 Soslow RA, Dannenberg AJ, Rush D, Woerner BM, Khan KN, Masferrer J, Koki AT: Cox-2 is expressed in human pulmonary, colonic, and mammary tumors. Cancer 2000;89:2637-2645.

16 Shirai Y, Fujita Y, Hashimoto R, Ohi K, Yamamori H, Yasuda Y, Ishima T, Suganuma H, Ushida Y, Takeda M, Hashimoto K: Dietary intake of sulforaphane-rich broccoli sprout extracts during juvenile and adolescence can prevent phencyclidine-induced cognitive deficits at adulthood. PLoS One 2015;10:e0127244.

17 Dwivedi S, Rajasekar N, Hanif K, Nath C, Shukla R: Sulforaphane ameliorates okadaic acid-induced memory impairment in rats by activating the nrf2/ho-1 antioxidant pathway. Mol Neurobiol DOI:10.1007/s12035015-9451-4.

18 Lee S, Kim J, Seo SG, Choi BR, Han JS, Lee KW, Kim J: Sulforaphane alleviates scopolamine-induced memory impairment in mice. Pharmacol Res 2014;85:23-32.

19 Dash PK, Zhao J, Orsi SA, Zhang M, Moore AN: Sulforaphane improves cognitive function administered following traumatic brain injury. Neurosci Lett 2009;460:103-107.

20 Zhu G, Liu Y, Wang Y, Bi X, Baudry M: Different patterns of electrical activity lead to long-term potentiation by activating different intracellular pathways. J Neurosci 2015;35:621-633.

21 Chen C, Wang Y, Zhang J, Ma L, Gu J, Ho G: Contribution of neural cell death to depressive phenotypes of streptozotocin-induced diabetic mice. Dis Model Mech 2014;7:723-730.

22 Xiang Q Zhang J, Li CY, Wang Y, Zeng MJ, Cai ZX, Tian RB, Jia W, Li XH: Insulin resistance-induced hyperglycemia decreased the activation of akt/creb in hippocampus neurons: Molecular evidence for mechanism of diabetes-induced cognitive dysfunction. Neuropeptides 2015;54:9-15.

23 Adamiec-Mroczek J, Zajac-Pytrus H, Misiuk-Hojlo M: Caspase-dependent apoptosis of retinal ganglion cells during the development of diabetic retinopathy. Adv Clin Exp Med 2015;24:531-535.

24 Yonguc GN, Dodurga Y, Adiguzel E, Gundogdu G, Kucukatay V, Ozbal S, Yilmaz I, Cankurt U, Yilmaz Y, Akdogan I: Grape seed extract has superior beneficial effects than vitamin e on oxidative stress and apoptosis in the hippocampus of streptozotocin induced diabetic rats. Gene 2015;555:119-126.

25 Zhou X, Zhang F, Hu X, Chen J, Wen X, Sun Y, Liu Y, Tang R, Zheng K, Song Y: Inhibition of inflammation by astaxanthin alleviates cognition deficits in diabetic mice. Physiol Behav 2015;151:412-420.

26 O'Connell MA, Hayes JD: The keap1/nrf2 pathway in health and disease: From the bench to the clinic. Biochem Soc Trans 2015;43:687-689.

27 Zhu G, Li J, He L, Wang X, Hong X: Mptp-induced changes in hippocampal synaptic plasticity and memory are prevented by memantine through the bdnf-trkb pathway. Br J Pharmacol 2015;172:2354-2368.

28 Zhu G, Wang X, Wu S, Li Q: Involvement of activation of pi3k/akt pathway in the protective effects of puerarin against mpp+-induced human neuroblastoma sh-sy5y cell death. Neurochem Int 2012;60:400-408. 